BULL. AUSTRAL. MATH, SOC.

\title{
ON THE ADDITIVE GROUPS OF
}

\section{SUBDIRECTLY IRREDUCIBLE RINGS}

\author{
Yasuyuki Hirano and Isao Mogami
}

In this paper we study the additive group structure of subdirectly irreducible rings and their hearts. We give an example of a torsion-free, non-reduced abelian group which is not the underlying additive group of any associative subdirectly irreducible ring. It is a counterexample to a theorem in Feigelstock's book "Additive Groups of Rings."

\section{Introduction.}

In [1] and [2], s. Feigelstock studied the additive group structure of subdirectly irreducible rings, and the results of these papers are collected in \$ 4, Chapter 4 of [3]. Moreover, in [4], Feigelstock proved that if $R$ is a commutative subdirectly irreducible ring with heart $S$, then either $R$ is a field or $S^{2}=0$. In this paper, first of all, we attempt to extend this result to certain classes of non-commutative rings. That is, we shall prove that a subdirectly irreducible PI-ring (respectively a one-sided duo ring) with square-nonzero heart is a simple Artinian ring (respectively a division ring). However, an example in

Received 22 November 1985. Dedicated to Professor Akira Hattori on his 60 th birthday.

Copyright Clearance Centre, Inc. Serial-fee code: 0004-9727/86 $\$ A 2.00+0.00$. 
Jacobson's book [6] shows that a subdirectly irreducible ring with squarenonzero heart need not be simple in general. As a corollary, we determine the additive group structure of those rings. Next we shall give a complete classification of the additive groups of the hearts of subdirectly irreducible rings. Using it we shall also give a necessary condition for an abelian group $G$ to be the additive group of some subdirectly irreducible xing with square-nonzero heart. Finally, we shall give a counterexample to Feigelstock [3, Theorem 4.4.4] : A torsion-free abelian group $G$ is the additive group of some associative subdirectly irreducible ring if and only if $G$ is not reduced. But, in general, the if part of this assertion is not true. In fact, the group $Q^{+} \oplus Z$ is a counterexample to the above assertion. Therefore the classification of the torsion-free additive groups of associative subdirectly irreducible rings is not complete.

\section{Notation and terminology.}

Throughout this paper, $R$ will denote an associative ring (possibly without 1). The additive group of $R$ is denoted by $R^{+}$, in particular, $Q^{+}$means the full rational group. The quasi-cyclic (that is $p$-Prüfer) group will be denoted by $Z\left(p^{\infty}\right)$, and a cyclic group of order $m$, by $2(m)$. A ring $R$ is said to be subdirectly irreducible if the intersection $S$ of all its nonzero ideals is not 0 . In this case, the

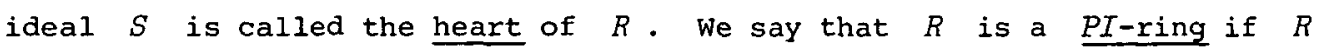
satisfies a polynomial identity with coefficients in the centroid and at least one coefficient is invertible. A ring $R$ is called right duo (respectively left duo) if every right (respectively left) ideal of $R$ is two-sided. A ring $R$ is called a finite-chain ring if the lattice of ideals of $R$ forms a finite chain. Following Feigelstock [3], an abelian group $G$ is said to be a subdirectly irreducible ring group if there exists an (associative) subdirectly irreducible ring $R$ such that $R^{+}=G$.

\section{Main results.}

We begin our study by proving the following theorem which is a generalization of Feigelstock [4, Theorem 4]. 
THEOREM 1. Let $R$ be a subdirectly irreducible ring with squarenonzero heart $S$. Then the following hold:

(1) If $R$ is a PI-ring, then $R$ is a simple Artinian ring.

(2) If $S$ is a right (or left) duo ring, then $R$ is a division ring. In particular, if $R$ is a right (or left) duo ring, then $R$ is a division ring.

Proof. First we claim that in general, the square-nonzero heart $S$ is a simple ring. If the left annihilator $\ell(S)=\{r \in R \mid r s=0$ for all $s \in S\}$ of $S$ is a nonzero ideal, then $S \subseteq \ell(S)$. This implies that $S^{2} \subseteq \ell(S) S=0$, which contradicts our assumption. Therefore we have $\ell(S)=0$. Similarly we have the right annihilator $r(S)=0$. Thus, for any nonzero $a \in S$, SaS is a nonzero ideal of $R$. Since $R$ is subdirectly irreducible, we conclude $S a S=S$. This means that $S$ is a simple ring.

(1) If $R$ satisfies a polynomial identity, then by [8, Theorem 2 and nddendum], $S$ has a non-trivial centre $C$. Since $S$ is simple, $C$ must be a field. Thus $S$ has an identity element $e$. Let $r$ be an arbitrary element of $R$. Then we have $S(r-e r)=0$. Since $h(S)=0$, we have $r=e r \in S$ for all $r \in R$. Therefore $R$ equals $S$, and hence $R$ is a simple ring with identity. By Kaplansky's theorem [7, Theorem 1], $R$ is finite dimensional over its centre. In particular, $R$ is Artinian.

(2) Since the heart $S$ is a simple right (or left) duo ring, it is easy to see that $S$ is a division ring. Now, the rest of the proof proceeds in the same way as in the proof of (1).

As a direct consequence of Theorem 1, we obtain the following

COROLLARY 2. Let $R$ be a PI-ring or a one-sided duo ring. If $R$ is a finite-chain ring, then every proper ideal of $R$ is nilpotent.

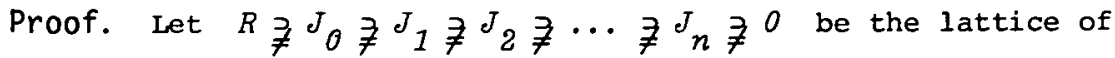
ideals of $R$. It suffices to show that $J_{0}$ is nilpotent. If $J_{n}^{2} \neq 0$, then by Theorem $1, R$ is a simple ring. This is a contradiction. Hence we have $J_{n}^{2}=0$. Similarly we have $J_{i}^{2} \subseteq J_{i+1}$ for all $0 \leq i \leq n-1$. Therefore we conclude that $J_{0}$ is nilpotent. 
The following example shows that if $R$ is not a PI-ring or a one-sided duo ring, then the assertions of Theorem 1 and Corollary 2 need not be true in general.

EXAMPLE 3. Let $M$ be an ${ }_{n}$-dimensional vector space over some field $D$. We consider the complete ring $R$ of linear transformations in $M$. Let $e$ be an infinite cardinal number not exceeding $\aleph_{n}$ and let $L_{e}$ denote the totality of linear transformations in $M$ of rank less than $e$. Then, by Jacobson [6, Theorem 1, p. 93], the ideals $R \not L_{n} \not L_{n-1} \not \cdots \not L_{\aleph_{0}} \not{ }^{0}$ are the only ideals. As is well known, $R$ is a von Neumann regular ring, and so every ideal of $R$ is idempotent.

The following is a generalization of [4, Corollary 5].

COROLLARY 4. Let $R$ be a subdirectly irreducible ming with squarenonzero heart $S$. If $R$ is a PI-ring or a one-sided duo ring, then either $R^{+}=\oplus_{\alpha} Q^{+}$or $R^{+}=\oplus_{B}^{\oplus} Z(p)$, where $p$ is some prime, $\alpha$ and $B$ are some cardinal numbers.

Proof. By Theorem $l R$ is a simple Artinian ring. Hence the centre $C$ of $R$ is a field. Thus our assertion follows from the fact that $R$ is a vector space over the prime field of $C$.

Next we shall determine the additive groups of the hearts of subdirectly irreducible rings.

THEOREM 5. Let $G$ be an abelian group. Then the following are equivalent:

(1) $G$ is the additive group of the heart of some subdirectly irreducible ring.

(2) Either $G=\underset{\alpha}{\oplus} Q^{+}$or $G=\underset{\beta}{\oplus} Z(p)$, where $p$ is some prime, $\alpha$ and $B$ are some cardinal numbers.

Proof. (1) $\Rightarrow(2)$. Let $S$ be the heart of a subdirectly irreducible ring. Let $p$ be a prime. Then either $p S=0$ or $p S=S$. If $p S=0$ for some prime $p$, then $S$ is a vector space over the field $G F(p)$, and hence $S^{+}=\underset{\beta}{\oplus} Z(p)$ for some cardinal $\beta$. On the other hand 
if $p S=S$ for all primes $p$, then $S^{t}$ is divisible. Hence, by $[5$,

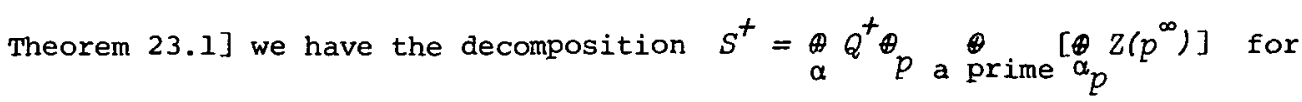
some cardinal numbers $\alpha, \alpha_{p}$. Let $n$ be an arbitrary nonzero integer. If the ideal $T_{n}=\{a \in R \mid n a=0\} \neq 0$, then $S \subseteq T_{n}$, and so $n S=0$. But this contradicts the divisibility of $S^{+}$. Thus, $S^{+}$is torsion-free in this case. Therefore we conclude that $S^{+}=\oplus Q^{+}$for some cardinal $\alpha$. (2) $\Longrightarrow$ (1). If $G=\underset{\alpha}{\oplus} Q^{+}$, then $G$ is the additive group of a field extension of degree $\alpha$ of $Q$. On the other hand, if $G=\underset{\beta}{(T)} Z(p)$, then $G$ is the additive group of a field extension of degree $B$ of the field $G F(p)$. This completes the proof.

Next we shall describe the additive group structure of a subdirectly irreducible ring with square-nonzero heart.

THEOREM 6. Let $R$ be a subdirectly irreducible ring with squarenonzero heart $S$. Then either $R^{+}$is torsion-free, non-reduced or $R^{+}=\oiint_{\alpha} Z(p)$ for some prime $p$ and some cardinal number $\alpha$.

Proof. First we consider the case that $R^{+}$is torsion-free. In this case, by Theorem 5, we have $S^{+}=\underset{\beta}{\oplus} Q^{+}$for some cardinal $\beta$. Therefore $R^{+}$is not reduced.

Next, assume that $R^{+}$is not torsion-free. Then, clearly, the heart is contained in the ideal consisting of all torsion elements in $R$. Again by Theorem 5 we obtain $S^{+}=\underset{\gamma}{\oplus} 2(p)$ for some prime $p$ and some cardinal $\gamma$. Now we claim that $p R=0$. If $p R \neq 0$, then $S \subseteq p R$, and so $S^{2} \subseteq S(p R)=0$. This is a contradiction. Thus $R$ can be considered as a vector space over the field $G F(p)$, and hence $R^{+}=\underset{\alpha}{\oplus} Z(p)$ for some cardinal $\alpha$.

If an abelian additive group $G$ has the form $\underset{\alpha}{\oplus} Z(p)$ for some prime $p$ and some cardinal number $\alpha$, then $G$ is the additive group of a field extension of degree $\alpha$ of the field $G F(p)$, and so $G$ is an 
associative aubdirectly irreducible ring group. In [3, Theorem 4.4.4], Feigelstock asserted that every torsion-free, non-reduced abelian group is an associative subdirectly irreducible ring group. But the following example shows that Feigelstock's assertion is not valid in general.

EXAMPLE 7. The additive group $Q^{+} \oplus 2$ is not an associative subdirectly irreducible ring group.

Proof. Suppose, to the contrary, that there exists an associative subdirectly irreducible ring $R$ such that $R^{+}=Q^{+} \oplus Z$. Let $S$ be the heart of $R$. Then we conclude that $S^{+}=Q^{+}(1,0)$, by Theorem 5 . Now put $e=(1,0)$ and $f=(0,1)$. Since $S=Q e$ is an ideal of $R$, we have $e^{2}=q_{1} e$, ef $=q_{2} e$ and $f e=q_{3} e$ for some $q_{1}, q_{2}, q_{3} \in Q$. By the associativity of $R$, we have $q_{1} q_{2} e=(e f) e=e(f e)=q_{1} q_{3} e$. If $q_{1} \neq 0$, then we obtain $q_{2}=q_{3}$. In this case, $R$ is a commutative subdirectly irreducible ring with square-nonzero heart $S$. But this is impossible by Corollary 4. Hence, we have $e^{2}=0$, and so $Z\left(q_{2}, q_{3}\right)$ is a nonzero ideal of $R$, where $2\left(q_{2}, q_{3}\right)$ denotes the subring of $Q$, generated by $q_{2}$ and $q_{3}$. Evidently, the ideal $z\left(q_{2}, q_{3}\right) e$ does not contain $S$. This contradicts the fact that $S$ is the heart of $R$. This completes the proof.

In view of Example 7, it seems that there is not a torsion-free, non-divisible, subdirectly irreducible ring group. However we have the following example. Therefore the following remains as an open problem: Characterize the torsion-free abelian groups which occur as the additive groups of associative subdirectly irreducible rings.

EXAMPLE 8. Again we consider the ring $R$ in Example 3, and we put $D=Q, L_{\aleph_{0}}=L$. Then clearly $L$ is a vector space over $Q$. Hence, $L^{+}=\underset{\alpha}{\oplus} Q^{+}$for some cardinal $\alpha$. Let $T$ be the subring of $R$ generated by $L$ and 1 . We claim that $T$ is a subdirectly irreducible ring such that $T^{+}=\left[\begin{array}{ll}\oplus & Q^{+}\end{array}\right] \oplus Z$ for some cardinal $\alpha$. Let $x$ be an arbitrary 
nonzero element of $T$. By the first part of the proof of Theoren 1 , we see that $L$ is a simple ring with $\ell(L)=r(L)=0$. Hence $L x L \neq 0$, and so $L x L=L$. This shows that $L$ is the heart of $T$. It is easy to see that $T^{+}=\left[\underset{\alpha}{\oplus} Q^{+}\right] \oplus Z$ for some cardinal $\alpha$.

\section{References}

[1] S. Feigelstock, "The additive groups of subdirectly irreducible rings", Bull. Austral. Math. Soc. 20 (1979), 164-170.

[2] S. Feigelstock, "The additive groups of subdirectly irreducible rings II", BulZ. Austral. Math. Soc. 22 (1980), 407-409.

[3] S. Feigelstock, Additive Groups of Rings, (Pitman, Boston-LondonMelbourne, 1983).

[4] S. Feigelstock, "A note on subdirectly irreducible rings", Buzl. Austraz. Math. Soc. 30 (1984), 137-141.

[5] L. Fuchs, Infinite Abelian Groups, Vol. I-II (Academic Press, New York-London, 1970 and 1973).

[6] N. Jacobson, Stmucture of Rings, (Amer. Math. Soc. Colloquium Publ. Vol. 37, Rev. ed. Providence, R.I.: 1964).

[7] I. Kaplansky, "Rings with a polynomial identity", Bulz. Amer. Math. Soc. 54 (1948), 575-580.

[8] L. Rowen, "Some results on the centre of a rings with polynomial identity", Bull. Amer. Math. Soc. 79 (1973), 219-223.

Department of Mathematics

Okayama University

Okayama 700

JAPAN

\author{
Tsuyama College of Technology \\ Tsuyama 708 \\ and \\ Department of Mathematics \\ Okayama University \\ Okayama 700 \\ JAPAN
}

